Dhaka Univ. J. Sci. 60(2): 217-222, 2012 (July)

\title{
Algorithm to Perform a Complete RHS Parametric Analysis for LPP with Bounded Variables
}

\author{
Sanwar Uddin Ahmad", M. Babul Hasan and M. Ainul Islam \\ Department of Mathematics, University of Dhaka, Dhaka-1000, Bangladesh
}

Received on 18. 8. 11. Accepted for Publication on 15. 12.11

\begin{abstract}
Linear Programming problem (LPP)s with upper bounded variables can be solved using the Bounded Simplex method, without the explicit consideration of the upper bounded constraints. One can consider the upper bounded constraints explicitly and perform the regular righthand-side parametric analysis of LPPs with bounded variables. This paper develops a method to perform the parametric analysis where the upper bounded constraints are considered implicitly, thus reduce the size of the basis matrix.
\end{abstract}

Keywords. Linear Programming, bounded variable, parametric programming.

\section{Introduction}

Consider the LP problem with upper bounded variables

$$
\begin{array}{ll}
\text { Max } & \mathrm{z}=c^{T} x \\
\text { s.t. } & A x \leq b \\
& 0 \leq x \leq U
\end{array}
$$

These problems have been solved by using the upper bound simplex technique without increasing the size of the problem (see: Murty ${ }^{1}$, Dantzig $^{2}$ ). Wagner ${ }^{3}$ developed the dual simplex method for LP with bounded variables, which is further studied by $\operatorname{Maros}^{4,5}$.

However the data obtained to estimate the parameter values $(A, c, b, U)$ used in the above model often are rather crude or nonexistent, so that the parameters in the original formulation may represent deliberate overestimates or underestimates to protect the interests of the estimators. For these reason it is important to perform a parametric analysis to investigate the effects on the optimal solution provided by the simplex method (SM) if the parameters take on other possible values. The traditional way to evaluate any imprecision in the parameters of an LP model is through a post-optimization analysis, with the help of sensitivity analysis, shadow prices and parametric programming. The method of sensitivity analysis in SM is well developed on the foundation of optimal basis, it requires little computational effort, has been introduced in numerous papers and text books so far (see, for example: Dantzig ${ }^{2,6}$, $\left.\mathrm{Gal}^{7}\right)$ and used in many LP codes.

In this paper we introduced a parameter $\theta$ in the right hand side vector $b$ of (1). This parametric demand has practical significance since the uncertainty in demand requirements is commonly seen in locational decision making environments. This paper presents an algorithm to perform the parametric analysis of the above problem.
Panwalkar $^{8}$ established a method for the parametric analysis of LP problems with upper bounded variables, where the upper bounds in (1) have been parametrized as $0 \leq x \leq U+\theta V$, that provides an approximation to the maximum value of the parameter $\theta$ maintaining the feasibility of the problem. Later Dahiya and Verma ${ }^{9}$ discussed the positive sensitivity analysis (PSA) in LP with bounded variables within which the components of a given optimal solution which are strictly between their bounds remain strictly between bounds and which are at their lower and upper bounds remain at lower and upper bounds respectively.

In this paper, we consider the parametric programming where the demand is allowed to vary as a linear function of the parameter $\theta$ within the given bounds of the variables. An algorithm has been established that will find all possible optimal solutions as a linear function of the parameter. Finally a numerical example is provided.

\section{RHS Parametric Analysis}

Consider the LP problem with bounded variables

$$
\operatorname{Max} z=c^{T} x
$$

Subject to $A x=b+\theta b^{\prime}$

$$
0 \leq x \leq U
$$

where $c, x, U \in \mathfrak{R}^{n}, b \in \mathfrak{R}^{m}, A=\left[a_{i j}\right]_{m \times n}$ is a matrix of rank $m . U$ is the vector of the upper bounds of the decision variables and $\theta$ is a real valued parameter. Let $c_{j}$ be the $j^{\text {th }}$ component of the vector $c, A_{j}$ be the $j^{\text {th }}$ activity vector i.e. the column of the coefficient matrix in the simplex tableau corresponding to the variable $x_{j}, B$ and $N$ denote the submatrix of $A$ in the simplex tableau corresponding to the basic $\left(x_{B}\right)$ and non-basic $\left(x_{N}\right)$ variables respectively. Let $\left(U_{B}\right)_{i}$ be the upper bound of the $i^{\text {th }}$ basic variable $\left(x_{B}\right)_{i}$. Let

*Correspondence author : E-mail: sanwar@univdhaka.edu 
$J=\{1,2, \cdots, n\}$,

$I=\left\{i \in J \mid x_{i}\right.$ is a basic variable $\}$. It is assumed that problem (2) has an optimal solution for $\theta=0$. Then the optimum simplex tableau of (2) for $\theta=0$ has the form,

$$
\begin{aligned}
& z=c_{B}^{T} x_{B}+c_{N}^{T} x_{N}, \\
& B x_{B}+N x_{N}=b
\end{aligned}
$$

Any variable that reaches at its upper bound, is replaced by $x_{i}^{\prime}=u_{i}-x_{i}$, thus all the $m$ basic variables are nonnegative and the remaining $(n-m)$ non-basic variables are at zero level. To establish the method for the parametric analysis we need the following result.

Theorem. Consider the problem described in (2). If $\theta$ is any real valued parameter then the solution obtained for $\theta=0$ will remain optimal for $\theta_{L} \leq \theta \leq \theta_{U}$, where $\theta_{L}=\max \left\{\theta_{L_{1}}, \theta_{L_{2}}\right\}, \quad \theta_{U}=\min \left\{\theta_{U_{1}}, \theta_{U_{2}}\right\}$, $\theta_{L_{1}}=\max _{i}\left\{\frac{-\left(B^{-1} b\right)_{i}}{\left(B^{-1} b^{\prime}\right)_{i}} \mid\left(B^{-1} b^{\prime}\right)_{i}>0\right\}$,

$\theta_{L_{2}}=\max _{i}\left\{\frac{\left(U_{B}\right)_{i}-\left(B^{-1} b\right)_{i}}{\left(B^{-1} b^{\prime}\right)_{i}} \mid\left(B^{-1} b^{\prime}\right)_{i}<0\right\}$,

$\theta_{U_{1}}=\min _{i}\left\{\frac{-\left(B^{-1} b\right)_{i}}{\left(B^{-1} b^{\prime}\right)_{i}} \mid\left(B^{-1} b^{\prime}\right)_{i}<0\right\}$,

$\theta_{U_{2}}=\min _{i}\left\{\frac{\left(U_{B}\right)_{i}-\left(B^{-1} b\right)_{i}}{\left(B^{-1} b^{\prime}\right)_{i}} \mid\left(B^{-1} b^{\prime}\right)_{i}>0\right\}$.

Proof. For any real valued parameter $\theta$ the $i^{\text {th }}$ basic variable $\left(x_{B}\right)_{i}$ can be written using equation (4) as,

$$
\left(x_{B}\right)_{i}=\left(B^{-1} b\right)_{i}+\theta\left(B^{-1} b^{\prime}\right)_{i}+\sum_{j \in J \mid I} P_{j} x_{j}
$$

where $P_{j}=B^{-1} N$

Since the dual solution of (2) does not depend on $\theta$, therefore it remains dual feasible for any $\theta$. Thus if $x_{j}$ in (5) remain non-basic, then the feasibility of the problem remains unaffected i.e. the solution (5) will remain optimum only if,

$$
0 \leq\left(x_{B}\right)_{i}=\left(B^{-1} b\right)_{i}+\theta\left(B^{-1} b^{\prime}\right)_{i} \leq\left(U_{B}\right)_{i}
$$

The non-negativity condition of $\left(x_{B}\right)_{i}$ is satisfied if,

$$
\theta \geq \frac{-\left(B^{-1} b\right)_{i}}{\left(B^{-1} b^{\prime}\right)_{i}}, \text { for }\left(B^{-1} b^{\prime}\right)_{i}>0
$$

and $\quad \theta \leq \frac{-\left(B^{-1} b\right)_{i}}{\left(B^{-1} b^{\prime}\right)_{i}}$, for $\left(B^{-1} b^{\prime}\right)_{i}<0$

The condition $\left(x_{B}\right)_{i} \leq\left(U_{B}\right)_{i}$ is satisfied if,

$\theta \leq \frac{\left(U_{B}\right)_{i}-\left(B^{-1} b\right)_{i}}{\left(B^{-1} b^{\prime}\right)_{i}}$, for $\left(B^{-1} b^{\prime}\right)_{i}>0$

and

$\theta \geq \frac{\left(U_{B}\right)_{i}-\left(B^{-1} b\right)_{i}}{\left(B^{-1} b^{\prime}\right)_{i}}$, for $\left(B^{-1} b^{\prime}\right)_{i}<0$

Now combining (7) and (10) let us define

$$
\begin{aligned}
\theta_{L_{1}} & =\max _{i}\left\{\frac{-\left(B^{-1} b\right)_{i}}{\left(B^{-1} b^{\prime}\right)_{i}} \mid\left(B^{-1} b^{\prime}\right)_{i}>0\right\}, \\
\theta_{L_{2}} & =\max _{i}\left\{\frac{\left(U_{B}\right)_{i}-\left(B^{-1} b\right)_{i}}{\left(B^{-1} b^{\prime}\right)_{i}} \mid\left(B^{-1} b^{\prime}\right)_{i}<0\right\} \text { and } \\
\theta_{L} & =\max \left\{\theta_{L_{1}}, \theta_{L_{2}}\right\} .
\end{aligned}
$$

Similarly using (8) and (9) let us define $\theta_{U_{1}}=\min _{i}\left\{\frac{-\left(B^{-1} b\right)_{i}}{\left(B^{-1} b^{\prime}\right)_{i}} \mid\left(B^{-1} b^{\prime}\right)_{i}<0\right\}$,

$\theta_{U_{2}}=\min _{i}\left\{\frac{\left(U_{B}\right)_{i}-\left(B^{-1} b\right)_{i}}{\left(B^{-1} b^{\prime}\right)_{i}} \mid\left(B^{-1} b^{\prime}\right)_{i}>0\right\} \quad$ and
$\theta_{U}=\min \left\{\theta_{U_{1}}, \theta_{U_{2}}\right\}$. Thus $\theta_{L}$ and $\theta_{U}$ become the lower and upper bound for $\theta$ respectively, for which the solution remain optimum, hence the result.

Remark1. If all $\left(B^{-1} b^{\prime}\right)_{i} \leq 0$, then let $\theta_{L_{1}}=-\infty$. Also if corresponding to every $\left(B^{-1} b^{\prime}\right)_{i}<0$, the basic variable $\left(x_{B}\right)_{i}$ does not have a finite upper bound, then $\theta_{L_{2}}=-\infty$. 
Remark2. If all $\left(B^{-1} b^{\prime}\right)_{i} \geq 0$, then let $\theta_{U_{1}}=\infty$. Also if corresponding to every $\left(B^{-1} b^{\prime}\right)_{i}>0$, the basic variable $\left(x_{B}\right)_{i}$ does not have a finite upper bound, then $\theta_{U_{2}}=\infty$ Gass and Saaty ${ }^{10,11}$ showed that there are a finite number of such characteristic values.

In general if $\theta_{L}^{k}$ represent the $k^{\text {th }}$ characteristic value of $\theta$, then the lower bound at the $k^{\text {th }}$ stage is obtained as,

$$
\begin{aligned}
\theta_{L_{1}}^{k} & =\max _{i}\left\{\frac{-\left(B^{-1} b\right)_{i}^{k}}{\left(B^{-1} b^{\prime}\right)_{i}^{k}} \mid\left(B^{-1} b^{\prime}\right)_{i}^{k}>0\right\} \\
\theta_{L_{2}}^{k} & =\max _{i}\left\{\frac{\left(U_{B}\right)_{i}^{k}-\left(B^{-1} b\right)_{i}^{k}}{\left(B^{-1} b^{\prime}\right)_{i}^{k}} \mid\left(B^{-1} b^{\prime}\right)_{i}^{k}<0\right\}
\end{aligned}
$$

and $\theta_{L}^{k}=\max \left\{\theta_{L_{1}}^{k}, \theta_{L_{2}}^{k}\right\}$.

In (11) and (12) the notation ()$_{i}^{k}$ denotes the $i^{\text {th }}$ term in the column vector ( ) at the $k^{\text {th }}$ iteration and the optimum solution at $k^{\text {th }}$ stage is given by

$x_{B}^{k}=\left(B^{k}\right)^{-1}\left[b^{k}+\theta b^{k}\right], \theta_{L}^{k} \leq \theta \leq \theta_{L}^{k-1}$

Similarly if $\theta_{U}^{m}$ represent the $m^{\text {th }}$ characteristic value of $\theta$, then the upper bound at the $m^{\text {th }}$ stage is obtained as,

$\theta_{U_{1}}^{m}=\min _{i}\left\{\frac{-\left(B^{-1} b\right)_{i}^{m}}{\left(B^{-1} b^{\prime}\right)_{i}^{m}} \mid\left(B^{-1} b^{\prime}\right)_{i}^{m}<0\right\}$

$\theta_{U_{2}}^{m}=\min _{i}\left\{\frac{\left(U_{B}\right)_{i}^{m}-\left(B^{-1} b\right)_{i}^{m}}{\left(B^{-1} b^{\prime}\right)_{i}^{m}} \mid\left(B^{-1} b^{\prime}\right)_{i}^{m}>0\right\}$

and $\theta_{U}^{m}=\min \left\{\theta_{U_{1}}^{m}, \theta_{U_{2}}^{m}\right\}$.

The optimum solution at $m^{\text {th }}$ stage is given by (13) for $\theta_{U}^{m-1} \leq \theta \leq \theta_{U}^{m}$.

For problems without upper bounds the new basis can be obtained by carrying out a simplex iteration. For problems with upper bounds the algorithm for performing the parametric analysis is described next.

\section{Algorithm}

Assume that the LP problem (2) with bounded variables provides an optimum solution for $\theta=0$.

Step1. Solve problem (2) for $\theta=0$ using bounded simplex or simplex method and obtain the optimum table.

Step2. Using theorem 1, determine the characteristic values for $\theta$ i.e. $\theta_{L}^{k}$ and $\theta_{U}^{k}$ for $k=1$, such that the optimum solution obtained in step1 remains optimum.

Step3. For $\theta<0$, let $\theta_{L}^{k}=\theta_{L}$ denote the characteristic value for the lower bound of $\theta$ i.e. the value of $\theta$ at which the basis for optimum solution changes.

IF $\theta_{L}^{k}=-\infty$, there is no characteristic value for the problem. The basis at $\theta=0$ will remain optimum for all $\theta \leq \theta_{U}^{k}$ and GOTO Step 5.

ELSE If $\theta_{L}^{k}$ is finite valued, then we have the following two cases.

Case I. IF $\theta_{L}^{k}=\theta_{L_{1}}^{k}$, for $i=r$ (say) $\in I$.

THEN $x_{r}$ becomes non-basic and a dual simplex iteration is carried out to obtain the new basis as well as the new optimum solution since for $\theta<\theta_{L}^{k}$ the basic variable $x_{r}$ becomes negative and GOTO Step4.

IF the dual simplex iteration cannot be carried out THEN there is no feasible solution for $\theta<\theta_{L}^{k}$ and GOTO Step 5.

Case II. IF $\theta_{L}^{k}=\theta_{L_{2}}^{k}$, for $i=m$ (say) $\in I$.

THEN $x_{m}$ becomes non-basic at its upper bound as for $\theta<\theta_{L}^{k}$ the value of $x_{m}$ violates the upper bound constraint. Substitute $x_{m}=u_{m}-x_{m}^{\prime}$ and obtain the new basis presenting a new optimum solution by carrying out a simplex iteration GOTO Step4.

Step4. For $k=k+1$, determine the new characteristic values of $\theta$ and the corresponding optimum solutions using (11-13) and repeat Step 3-4.

Step5. For $\theta>\theta_{U}^{m}>0$, where $\theta_{U}^{m}=\theta_{U}^{1}$ is obtained in Step 2, the parametric analysis is performed as follows. 
Step6. IF $\theta_{U}^{m}=\infty$, THEN there is no characteristic value for the upper bound of $\theta$. The basis at $\theta=0$ will remain optimum for all $\theta \geq \theta_{L}^{m}$ and the analysis stopped.

ELSEIF $\theta_{U}^{m}$ is finite valued, then we have the following two cases.

Case I.

IF $\theta_{U}^{m}=\theta_{U_{1}}^{m}$, for $i=r$ (say) $\in I$ THEN $x_{r}$ becomes non-basic and a dual simplex iteration is carried out to obtain the new basis as well as the new optimum solution GOTO Step 7.

IF the dual simplex iteration cannot be carried out then there is no feasible solution for $\theta>\theta_{U}^{m}$ and the analysis stopped at this stage.

\section{Case II.}

IF $\theta_{U}^{m}=\theta_{U_{2}}^{m}$, for $i=n$ (say) $\in I$ THEN $x_{n}$ becomes non-basic at its upper bound, substitute $x_{n}=u_{n}-x_{n}^{\prime}$ and obtain the new basis presenting a new optimum solution by carrying out a simplex iteration and GOTO Step 7.

Step7. For $m=m+1$ evaluate the characteristic values for $\theta$ as well as the corresponding optimum solutions using (13-15) and repeat Step 6-7.

Stop.

\section{Numerical Example}

Consider the problem

$$
\operatorname{Max} \quad z=3 x_{1}+5 x_{2}+2 x_{3}
$$

Subject to

$$
\begin{gathered}
x_{1}+x_{2}+2 x_{3} \leq 10-\theta \\
2 x_{1}+4 x_{2}+3 x_{3} \leq 16+2 \theta \\
0 \leq x_{1} \leq 4,0 \leq x_{2} \leq 3,0 \leq x_{3} \leq 3
\end{gathered}
$$

Step1. At $\theta=0$ the optimum solution can be obtained easily and the optimum table is

Table. 1.

\begin{tabular}{|l|l|l|l|l|}
\hline & $x_{1}^{\prime}$ & $x_{3}$ & $x_{5}$ & Solution \\
\hline$x_{4}$ & $-1 / 2$ & $5 / 4$ & $-1 / 4$ & 4 \\
\hline$x_{2}$ & $-1 / 2$ & $3 / 4$ & $1 / 4$ & 2 \\
\hline & $1 / 2$ & $7 / 4$ & $5 / 4$ & 22 \\
\hline
\end{tabular}

The basic variables in the tableau are $x_{4}=4, x_{2}=2$ and all the non-basic variables $x_{1}^{\prime}, x_{3}, x_{5}$ are at zero level. The value of $x_{1}$ is easily obtained as $x_{1}=u_{1}-x_{1}^{\prime}=4$. Thus $x_{1}$ is at its upper bound and the value of the objective $z=22$

Step2. Now $B=\left(\begin{array}{ll}1 & 1 \\ 0 & 4\end{array}\right), B^{-1}=\left(\begin{array}{cc}1 & -1 / 4 \\ 0 & 1 / 4\end{array}\right), b^{\prime}=\left(\begin{array}{c}-1 \\ 2\end{array}\right)$. So $B^{-1} b^{\prime}=\left(\begin{array}{c}-3 / 2 \\ 1 / 2\end{array}\right)$.

Therefore $\theta_{L_{1}}^{1}=\max \left\{\frac{-2}{1 / 2}\right\}=-4$, for $i=2, \theta_{L_{2}}^{1}=-\infty$ and $\quad \theta_{U_{1}}^{1}=\min \left\{\frac{-4}{-3 / 2}\right\}=8 / 3, \quad$ for $i=4$, $\theta_{U_{2}}^{1}=\min \left\{\frac{3-2}{1 / 2}\right\}=2$, for $i=2$. Thus $\theta_{L}^{1}=\max \left\{\theta_{L_{1}}^{1}, \theta_{L_{2}}^{1}\right\}=-4=\theta_{L_{1}}^{1}$ with $i=2$ and $\theta_{U}^{1}=\min \left\{\theta_{U_{1}}^{1}, \theta_{U_{2}}^{1}\right\}=2=\theta_{U_{2}}^{1}$, with $i=2$. Hence the optimum solution of the problem is given by $x=B^{-1}\left(b+\theta b^{\prime}\right)=\left(\begin{array}{c}4 \\ 2+\theta / 2 \\ 0\end{array}\right), \quad z=22+\frac{5}{2} \theta, \quad$ for $\theta_{L}^{1} \leq \theta \leq \theta_{U}^{1}$, where $\theta_{L}^{1}=-4, \quad \theta_{U}^{1}=2$ are the first characteristic values of the lower and upper bound of $\theta$ respectively for which the solution is optimum.

Step3. $\theta_{L}^{1}=-4=\theta_{L_{1}}$ with $i=2$, thus $x_{2}$ becomes nonbasic and substituted by $x_{1}^{\prime}=u_{1}-x_{1}$ and the optimum table for $\theta<\theta_{L_{1}}$ is obtained by carrying out a dual simplex iteration as

Table. 2.

\begin{tabular}{|l|l|l|l|l|}
\hline & $x_{2}$ & $x_{3}$ & $x_{5}$ & Solution \\
\hline$x_{4}$ & -1 & $1 / 2$ & $-1 / 2$ & 2 \\
\hline$x_{1}$ & 2 & $3 / 2$ & $1 / 2$ & 8 \\
\hline & 1 & $5 / 2$ & $3 / 2$ & 24 \\
\hline
\end{tabular}

Step4. Using (12-14) for $k=2$, we obtained $\theta_{L}^{2}=\max \left\{\theta_{L_{1}}^{2}, \theta_{L_{2}}^{2}\right\}=-8=\theta_{L_{1}}^{2}$ with $i=1$. The optimum 
solution of the problem is $x=\left(\begin{array}{c}8+\theta \\ 0 \\ 0\end{array}\right), z=24+3 \theta$, for $\theta_{L}^{2} \leq \theta \leq \theta_{L}^{1}$, where $\theta_{L}^{2}=-8$. Now $\theta_{L}^{2}=\theta_{L_{1}}$ for $i=1$, as the dual simplex method cannot be applied therefore the analysis stopped at this stage.

Step5-6. From Table 1 , since $\theta_{U}^{1}=\theta_{U_{2}}^{1}$ for $i=2$, therefore $x_{2}$ becomes non-basic at its upper bound for $\theta>\theta_{U}^{2}$, substituted by $x_{2}=u_{2}-x_{2}^{\prime}$ and the new optimum basis is obtained by carrying out a simplex iteration as

Table. 3.

\begin{tabular}{|l|l|l|l|l|}
\hline & $x_{1}^{\prime}$ & $x_{2}^{\prime}$ & $x_{5}$ & Solution \\
\hline$x_{4}$ & $1 / 3$ & $5 / 3$ & $-2 / 3$ & $17 / 3$ \\
\hline$x_{3}$ & $-2 / 3$ & $-4 / 3$ & $1 / 3$ & $-4 / 3$ \\
\hline & $5 / 3$ & $7 / 3$ & $2 / 3$ & $-8 / 3$ \\
\hline
\end{tabular}

Step7. Using $(14-16)$ for $m=2$, we obtained $\theta_{U}^{2}=\min \left\{\theta_{U_{1}}^{2}, \theta_{U_{2}}^{2}\right\}=17 / 7=\theta_{U_{1}}^{2}$, with $i=4$. The optimum solution of the problem is given by $x=\left(\begin{array}{c}4 \\ 3 \\ -4 / 3+2 \theta / 3\end{array}\right), \quad z=\frac{73}{3}+\frac{4}{3} \theta, \quad$ for $\theta_{U}^{1} \leq \theta \leq \theta_{U}^{2}$, where $\theta_{U}^{2}=17 / 7$.

Following the same procedure described in the algorithm we obtained the following optimum solutions.

Table. 4.

\begin{tabular}{|l|l|l|l|l|}
\hline & $x_{1}^{\prime}$ & $x_{2}^{\prime}$ & $x_{4}$ & Solution \\
\hline$x_{5}$ & $-1 / 2$ & $-5 / 2$ & $-3 / 2$ & $-17 / 2$ \\
\hline$x_{3}$ & $-1 / 2$ & $-1 / 2$ & $1 / 2$ & $3 / 2$ \\
\hline & 2 & 4 & 1 & 3 \\
\hline
\end{tabular}

$x=\left(\begin{array}{c}4 \\ 3 \\ 3 / 2-\theta / 2\end{array}\right), z=30-\theta$, for $\theta_{U}^{2} \leq \theta \leq \theta_{U}^{3}$, where $\theta_{U}^{3}=3$.

Table. 5.

\begin{tabular}{|l|l|l|l|l|}
\hline & $x_{2}^{\prime}$ & $x_{3}$ & $x_{4}$ & Solution \\
\hline$x_{5}$ & -2 & -1 & -2 & -10 \\
\hline$x_{1}$ & -1 & 2 & 1 & 7 \\
\hline & 2 & 4 & 3 & 21 \\
\hline
\end{tabular}

$x=\left(\begin{array}{c}7-\theta \\ 3 \\ 0\end{array}\right), \quad z=26-3 \theta$, for $\theta_{U}^{3} \leq \theta \leq \theta_{U}^{4}$, where $\theta_{U}^{4}=7$.

Table. 6.

\begin{tabular}{|l|l|l|l|l|}
\hline & $x_{1}$ & $x_{3}$ & $x_{4}$ & Solution \\
\hline$x_{5}$ & -2 & -5 & -4 & -24 \\
\hline$x_{2}$ & 1 & 2 & 1 & 10 \\
\hline & 2 & 8 & 5 & 21 \\
\hline
\end{tabular}

$x=\left(\begin{array}{c}0 \\ 10-\theta \\ 0\end{array}\right), z=50-5 \theta$, for $\theta_{U}^{4} \leq \theta \leq \theta_{U}^{5}$, where $\theta_{U}^{5}=10$.

As the dual simplex method cannot be applied therefore the analysis stopped at this stage.

The optimum solutions thus obtained are given below

$$
\begin{gathered}
\max z=\left\{\begin{array}{l}
24+3 \theta, \quad-8 \leq \theta \leq-4 \\
22+5 \theta / 2, \quad-4 \leq \theta \leq 2 \\
73 / 3+4 \theta / 3, \quad 2 \leq \theta \leq 17 / 7 \\
30-\theta, \quad 17 / 7 \leq \theta \leq 3 \\
26-3 \theta, \quad 3 \leq \theta \leq 7 \\
50-5 \theta, \quad 7 \leq \theta \leq 10
\end{array}\right. \\
x=\left\{\begin{array}{l}
(8+\theta, 0,0), \quad-8 \leq \theta \leq-4 \\
(4,2+\theta / 2,0) \quad-4 \leq \theta \leq 2 \\
(4,3,-4 / 3+2 \theta / 3), \quad 2 \leq \theta \leq 17 / 7 \\
(4,3,3 / 2-\theta / 2), \quad 17 / 7 \leq \theta \leq 3 \\
(7-\theta, 3,0) \quad 3 \leq \theta \leq 7 \\
(0,10-\theta, 0) \quad 7 \leq \theta \leq 10
\end{array}\right.
\end{gathered}
$$

and

which is identical to the solutions obtained by the regular parametric analysis. 


\section{Conclusions}

In this paper an algorithm to perform the complete righthand side parametric analysis for LP problems with bounded variables is developed. The upper bound constraints are considered implicitly thus reduce the size of the basis matrix greatly and the computation become highly efficient than the regular parametric analysis. A numerical example has solved in this regard that clearly showed the efficiency of using the developed algorithm. Besides one can build a computer program to perform the parametric analysis using the same algorithm which is necessary for large scale problems.

1. Murty, K.G., 1976, Linear and combinatorial programming, John Wiley \& Sons INC., New York London Sydney Toronto.

2. Dantzig, G.B., 1963. Linear programming and extensions, Princeton University Press, Princeton, New Jersey.

3. Wagner, H. M., 1958, The Dual Simplex Algorithm for Bounded Variables, Naval Research Logistics Quarterly, 5, 257-261.
4. Maros, I., 2003a. A piecewise linear dual phase-I algorithm for the simplex method, Computational Optimization and Applications, 26, 63-81.

5. Maros, I., 2003b. A generalized dual phase-2 simplex algorithm, European Journal of Operational Research, 149, 1-16.

6. Dantzig, G. B., 1955. Upper Bounds, Secondary Constraints and Triangularity in Linear Programming, Vol 23, No. 2, Econometrica.

7. Gal, T., 1979. Postoptimal analysis, parametric programming and related topics, McGraw-Hill, NY.

8. Panwalkar, S. S., 1973, Parametric Analysis of Linear Programs with Upper Bounded Variables, Naval Research Logistics Quarterly, 20(1), 83 - 93.

9. Kalpana Dahiya and Vanita Verma, 2007. Positive Sensitivity Analysis In Linear Programming With Bounded Variables, ASOR Bulletin, 26(4), 2-26.

10. Gass, S. I. and T. L. Saaty, 1954. The Parametric Objective Function, Part I, Vol 2, Operations Research.

11. Gass, S. I. and T. L. Saaty, 1955. The Parametric Objective Function, Part II: Generalization, Vol 3, Operations Research. 